\title{
Alternative remedies for insomnia: a proposed method for personalized therapeutic trials
}

This article was published in the following Dove Press journal:

Nature and Science of Sleep

17 March 2017

Number of times this article has been viewed

\author{
Kate Romero ${ }^{1,2}$ \\ Balaji Goparaju',2 \\ Kathryn Russo',2 \\ M Brandon Westover' \\ Matt T Bianchi ${ }^{1,2}$ \\ 'Neurology Department, \\ Massachusetts General Hospital, \\ ${ }^{2}$ Division of Sleep Medicine, Harvard \\ Medical School, Boston, MA, USA
}

Correspondence: Matt T Bianchi

Wang 7 Neurology, Massachusetts

General Hospital, 55 Fruit Street, Boston,

MA 021 14, USA

$\mathrm{Tel}+\mathrm{I} 6177247426$

Fax +I 6I7 72465I3

Email mtbianchi@partners.org

\begin{abstract}
Insomnia is a common symptom, with chronic insomnia being diagnosed in 5-10\% of adults. Although many insomnia patients use prescription therapy for insomnia, the health benefits remain uncertain and adverse risks remain a concern. While similar effectiveness and risk concerns exist for herbal remedies, many individuals turn to such alternatives to prescriptions for insomnia. Like prescription hypnotics, herbal remedies that have undergone clinical testing often show subjective sleep improvements that exceed objective measures, which may relate to interindividual heterogeneity and/or placebo effects. Response heterogeneity can undermine traditional randomized trial approaches, which in some fields has prompted a shift toward stratified trials based on genotype or phenotype, or the so-called n-of-1 method of testing placebo versus active drug in within-person alternating blocks. We reviewed six independent compendiums of herbal agents to assemble a group of over 70 reported to benefit sleep. To bridge the gap between the unfeasible expectation of formal evidence in this space and the reality of common self-medication by those with insomnia, we propose a method for guided self-testing that overcomes certain operational barriers related to inter- and intraindividual sources of phenotypic variability. Patient-chosen outcomes drive a general statistical model that allows personalized self-assessment that can augment the open-label nature of routine practice. The potential advantages of this method include flexibility to implement for other (nonherbal) insomnia interventions.
\end{abstract}

Keywords: insomnia, over the counter, alternative remedy, herbal, supplement

\section{Introduction}

Insomnia is among the most common clinical complaints, with a symptom prevalence in adults exceeding 30\%, while more stringent diagnostic criteria place the prevalence of chronic insomnia in the $5-10 \%$ range. ${ }^{1}$ There are many options to consider for treating insomnia. Cognitive behavioral therapy for insomnia (CBT-I) is the long-recognized first-line approach to chronic insomnia, as indicated by the American Academy of Sleep Medicine (AASM) practice parameters. ${ }^{2}$ Although in-person CBT-I is not uniformly accessible, validated online options ${ }^{3-6}$ are increasingly available.

Although many chronic insomnia patients utilize prescription medications, adverse effects are well known and the risk-benefit balance remains a clinical challenge. ${ }^{7-9}$ In fact, there is extensive literature documenting the acute and chronic adverse effects associated with hypnotic drugs, including those approved by the Food and Drug Administration (FDA) and those used off-label. ${ }^{7,10-13}$ Certain hypnotics may objectively worsen sleep: benzodiazepines might worsen breathing (although the evidence 
is inconsistent ${ }^{14}$ ) and antidepressants might increase periodic limb movements ${ }^{15}$ or worsen breathing in some circumstances. ${ }^{16}$ The objective improvements in sleep metrics by polysomnography (PSG) are modest; a meta-analysis that included "z-drug" hypnotics for primary insomnia indicated only 12.9 minutes faster average onset latency (less with benzodiazepines or antidepressants) and only 11.4 minutes of average additional total sleep time (TST). ${ }^{17}$ Interestingly, subjective diary measures of latency and TST exceeded the objective metrics of benefit by $50-300 \%$. It is perhaps not surprising that the same trends are observed for natural remedies under the broad category of complementary and alternative medicine (CAM). Clinical trial data are sparse aside from two remedies, melatonin and valerian, which have been tested in several studies each. ${ }^{18-20}$ The outcomes are similar to a pattern commonly observed in trials of prescription hypnotics, in that subjective sleep improvements often exceeded the objective improvements. For example, a recent meta-analysis of 18 randomized control trials (RCTs) of valerian for insomnia found little objective improvement, despite clear subjective benefit. ${ }^{21}$

Recent reviews of the broader field of CAM therapy also report heterogeneous findings and emphasize methodological challenges. ${ }^{22,23}$ Despite these uncertainties, many adults turn to CAM remedies, ${ }^{24,25}$ with over one-third of adults reporting CAM use. ${ }^{26}$ Epidemiological surveys indicate that the use of CAM is common among those with insomnia ${ }^{27}(-5 \%$ reporting such use in 2002 National Health Interview Survey) as well as other systemic disorders that may be associated with disturbed sleep such as neurological conditions ${ }^{28}$ (44\% reporting CAM use in the 2007 National Health Interview Survey) and chronic medical conditions ${ }^{29}$ (17\% reporting mind-body therapies in the 2007 National Health Interview Survey). More recently, Bertisch et $\mathrm{al}^{30}$ analyzed the subset of the 2007 National Health Interview Survey participants who reported insomnia symptoms and found that nearly half indicated using CAM therapy.

When considering CAM therapy for insomnia, the limited evidence basis must be balanced against the possibility that interindividual heterogeneity, which compromises trial power, could be consistent with subsets who respond positively. This is well known in other fields and has prompted a shift toward stratified trials based on genotype or phenotype. The singlepatient extension of this idea is the so-called n-of-1 trial, which compares placebo against active drug in within-person alternating blocks. ${ }^{31,32}$ However, n-of-1 trials still require substantial resource investment and have not been consistently adopted. ${ }^{33}$ The placebo component restricts implementation of this otherwise highly personalized approach to research protocols, that is, one cannot take a placebo-based n-of-1 approach in clinical practice. In addition, patient-defined goals may not be captured by the experimental question of whether the intervention is statistically superior to placebo. To bridge the gap between formal prospective trials (group-wise or n-of-1) and the common patient-care practice of "open label" interventions with qualitative outcome assessment, we propose a simple method that leverages principles of trial design while remaining personalized.

\section{Methods}

We performed a manual search of several print and online resources concerning herbal and supplement remedies of potential relevance for insomnia: The Physician's Desk Reference for Herbal Medicines (PDR); ${ }^{34}$ the Encyclopedia of Herbal Medicine, ${ }^{35}$ The Drug and Natural Medicine Advisor (Time Life); ${ }^{36}$ Medline Plus (https://medlineplus.gov/ druginfo/herb All.html), the National Center for Complementary and Integrative Health Database (https://nccih.nih. gov/health/herbsataglance.htm), and the Natural Medicines online database (http://naturaldatabase.therapeuticresearch.

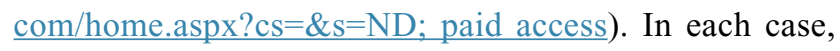
we manually searched for language such as "insomnia", "sleeplessness", or "disturbed sleep" as an indication for the remedy. We also searched for related terms that might be indirectly related to sleep effects ("sedative", "anxiety", "tranquilizer", "calming", and "nervousness"). Agents were included in the figures if any of the above six sources indicated insomnia as an indication, or, if none specifically listed insomnia, we accepted the agent if two or more of the sources listed a related indirect term (above). Agents listed in a resource but only for indications other than sleep are labeled as "misc" to indicate other uses, while agents not listed at all are given a blank ("-"). Most of the remedies listed for insomnia were also listed for other indications; we did not systematically assess this aspect. We did not consider indications such as antidepressant effects or treatment of pain. We also reviewed adverse effect listings from the PDR of Herbal Medicine and from UpToDate.

\section{Results and discussion Natural remedies for sleep}

An alphabetical list of herbal remedies is presented in Figures 1 and 2, with the relevant indications for each according to six sources ("Methods" section). The literature 


\begin{tabular}{|c|c|c|c|c|c|c|}
\hline Remedy & Medline Plus & $\mathrm{NIH} \mathrm{NCCIH}$ & $\begin{array}{c}\text { Natural } \\
\text { Medicines }\end{array}$ & $\begin{array}{l}\text { Encyc. of } \\
\text { Herbal Med }\end{array}$ & PDR & Time Life \\
\hline 5-HTP & Insomnia & - & Insomnia & - & Insomnia & - \\
\hline Albizia julibrissin & - & - & Insomnia & - & - & - \\
\hline Angelica archangelica & - & - & Insomnia & - & Misc & Misc \\
\hline Anise (Pimpinella) & - & - & Insomnia & Misc & Misc & Misc \\
\hline Ashwagandha & Insomnia & - & Insomnia & Insomnia & - & Misc \\
\hline Betony & - & - & Sedative & Sedative & - & - \\
\hline Bitter Orange (Neroli) & Misc & Misc & Insomnia & Sedative & Insomnia & Insomnia \\
\hline Black Horehound & - & - & Insomnia & Sedative & Sedative & - \\
\hline Black nightshade & - & - & Sedative & Sedative & Sedative & - \\
\hline Bog Bean & - & - & Misc & Misc & Insomnia & - \\
\hline Borage & - & Misc & Sedative & Misc & Sedative & - \\
\hline Bugleweed & - & - & Insomnia & Sedative & Insomnia & - \\
\hline Butterbur & - & Anxiety & Insomnia & - & Insomnia & - \\
\hline California Poppy & - & - & Insomnia & Insomnia & Insomnia & - \\
\hline Catnip (Nepeta) & Sedative & - & Insomnia & Sedative & Calming & Insomnia \\
\hline Catuaba & - & - & Insomnia & - & - & - \\
\hline Celery seed & - & - & Nervousness & Misc & Nervousness & - \\
\hline Chamomile (German) & - & Insomnia & Insomnia & Insomnia & Sedative & Anxiety \\
\hline Chaste Tree Berry & Misc & Misc & Insomnia & Insomnia & Insomnia & Misc \\
\hline Corn Poppy & - & - & Insomnia & Sedative & Insomnia & - \\
\hline Cowslip & - & - & Insomnia & Insomnia & Insomnia & - \\
\hline Cypress & - & - & Misc & Misc & Misc & Insomnia \\
\hline Dan-shen (Chinese red sage) & - & - & Insomnia & Insomnia & Sedative & - \\
\hline Dong Quai (Angelica sinensis) & Insomnia & - & Misc & Misc & Insomnia & Misc \\
\hline False Schisandra & - & - & - & - & Insomnia & - \\
\hline Fo-ti & - & - & Insomnia & Sedative & - & - \\
\hline Gardenia & - & - & Insomnia & Insomnia & - & Insomnia \\
\hline Ginkgo biloba & Misc & Misc & Insomnia & Misc & Anxiety & Misc \\
\hline Ginseng (Panax) & Misc & Misc & Insomnia & Insomnia & Insomnia & Misc \\
\hline Ginseng (Siberian) & Insomnia & - & Insomnia & - & - & Misc \\
\hline Gotu Kola & - & - & Anxiety & Anxiety & Misc & Misc \\
\hline Hawthorn & - & Misc & Anxiety & Misc & - & Insomnia \\
\hline Heather & - & - & Insomnia & Misc & Insomnia & - \\
\hline Hops & Insomnia & - & Insomnia & Insomnia & Insomnia & Insomnia \\
\hline Ignatia & - & - & - & - & - & Insomnia \\
\hline Jamaican Dogwood & - & - & Insomnia & Insomnia & Anxiety & - \\
\hline Jasmine & - & - & Misc & Calming & Misc & Anxiety \\
\hline
\end{tabular}

Figure I Natural remedies (alphabetical, A-J) listed by at least one of the six sources as possibly useful for sleep.

Notes: Green indicates direct reference to insomnia. Yellow indicates indirect terminologies. Gray indicates the resource mentioned the remedy for reasons other than sleep. "-" indicates the remedy was not mentioned in the resource.

Abbreviations: Encyc, encyclopedia; Misc, miscellaneous; PDR, Physician's Desk Reference for Herbal Medicines; NIH, National Institutes of Health; NCCIH, National Center for Complementary and Integrative Health Database.

concerning herbal and natural supplement agents is heterogeneous in several important respects.

First, the substances often have several names apparently interchanged as equivalent in the literature. For example, according to the Natural Medicines Database, Bugleweed is also reportedly known as Ajuga, Archangle, Ashangee, Chanvre d'Eau, Green Wolf's Foot, Gypsy Weed, Gypsywort, Hoarhound, Lycope, Lycope d'Amérique, Lycope d'Europe, Lycope de Virginie, Lycopi Herba, Lycopus Europea, Menta de Lobo, Patte-de-Loup, Paul's Betony, Sweet Bugle, Virginia
Water Horehound, Water Bugle, Water Horehound, and Wolfstrapp. By contrast, the Encyclopedia of Herbal Medicine lists only Gypsywort as a related species to Bugleweed. We did not conduct exhaustive cross-matching searches in this regard.

Second, different sources indicate different usages for the same agents. In some cases, agents were listed as helping insomnia in one source but as a stimulant in another source (peppermint and rhodiola). No single agent was listed as having a sleep or sleep-related usage in all six resources consulted. Most of the remedies were listed in 1-3 of the 


\begin{tabular}{|c|c|c|c|c|c|c|}
\hline Remedy & Medline Plus & $\mathrm{NIH} \mathrm{NCCIH}$ & $\begin{array}{l}\text { Natural } \\
\text { Medicines }\end{array}$ & $\begin{array}{l}\text { Encyc. of } \\
\text { Herbal Med }\end{array}$ & PDR & Time Life \\
\hline Jatamansi & - & - & - & - & Insomnia & - \\
\hline Kava & - & Insomnia & Insomnia & Insomnia & Insomnia & Insomnia \\
\hline Lavender (English) & Overdose & Insomnia & Insomnia & Insomnia & Insomnia & Insomnia \\
\hline Lemon Balm & - & - & Insomnia & Insomnia & Insomnia & Insomnia \\
\hline Lemon Verbena & - & - & Insomnia & Sedative & Insomnia & - \\
\hline Lemon-Wood (Schisandra) & - & - & - & - & Insomnia & - \\
\hline Linden & - & - & Insomnia & Sedative & Sedative & Insomnia \\
\hline Male Fern & - & - & Misc & - & Insomnia & - \\
\hline Meliltot (sweet clover) & - & - & Misc & Insomnia & Misc & - \\
\hline Mugwort & - & - & Insomnia & Misc & Insomnia & Systemic \\
\hline Nerve Root (Lady’s Slipper) & - & - & Insomnia & Insomnia & Insomnia & - \\
\hline Nutmeg (and Mace) & - & - & Insomnia & Insomnia & Insomnia & Insomnia \\
\hline Oats (Avena sativa) & Anxiety & - & Anxiety & Insomnia & Insomnia & Insomnia \\
\hline Pasque Flower & - & - & Insomnia & - & Insomnia & - \\
\hline Passion Flower & - & Insomnia & Insomnia & Insomnia & Insomnia & Insomnia \\
\hline Peppermint & - & Misc & Misc & Misc & Misc & Insomnia \\
\hline Poppyseed & - & - & Insomnia & - & Sedative & - \\
\hline Rauwolfia (serpentwood) & - & - & Insomnia & Insomnia & Insomnia & - \\
\hline Red-Spur Valerian & - & - & Sedative & - & Sedative & - \\
\hline Rehmannia (Chin. foxglove) & - & - & Misc & Misc & Insomnia & Insomnia \\
\hline Reishi Mushroom & Insomnia & - & Insomnia & - & - & - \\
\hline Rhodiola & Misc & Anxiety & Insomnia & Misc & Misc & - \\
\hline Saffron & - & - & Insomnia & Misc & Misc & Misc \\
\hline Schisandra (Wu-Wei-Zi) & Misc & Misc & Insomnia & Insomnia & Insomnia & Insomnia \\
\hline Senburi & - & - & - & - & Insomnia & - \\
\hline Skullcap & - & - & Insomnia & Insomnia & - & Insomnia \\
\hline St. John's Wort & - & Insomnia & Insomnia & Insomnia & Anxiety & Misc \\
\hline Sumbul & - & - & Sedative & - & Sedative & - \\
\hline Sweet Vernal Grass & - & - & Insomnia & - & Insomnia & - \\
\hline Sweet Violet & - & - & Insomnia & Insomnia & Insomnia & - \\
\hline Sweet Woodruff & - & - & Insomnia & Insomnia & Insomnia & - \\
\hline Tarragon & - & - & Sedative & Insomnia & - & Anxiety \\
\hline Valerian & - & Insomnia & Insomnia & Insomnia & Insomnia & Insomnia \\
\hline Water Hyssop & - & - & Nerve tonic & Misc & - & Anxiety \\
\hline Wild Lettuce & - & - & Insomnia & Insomnia & Tranquilizer & - \\
\hline Yarrow & - & - & Misc & Misc & Misc & Insomnia \\
\hline Ylang Ylang oil & - & - & Sedative & Sedative & - & Insomnia \\
\hline Zyzyphus (Da-Zao) & - & - & Sedative & Sedative & Sedative & - \\
\hline
\end{tabular}

Figure 2 Natural remedies (alphabetical, J-Z) listed by at least one of the six sources as possibly useful for sleep.

Notes: Green indicates direct reference to insomnia. Yellow indicates indirect terminologies. Gray indicates the resource mentioned the remedy for reasons other than sleep. "-" indicates the remedy was not mentioned in the resource.

Abbreviations: Encyc, encyclopedia; Misc, miscellaneous; PDR, Physician's Desk Reference for Herbal Medicines; NIH, National Institutes of Health; NCCIH, National Center for Complementary and Integrative Health Database.

six sources, and a small subset appeared in $4-5$ sources. A related issue is that the health benefits or indications may be reported differently across traditions, which were differentiated in some of the sources; we do not differentiate here.

Third, the terminology used for indications is nonstandardized and at times ill-defined from a medical standpoint, such as "nervous disorders", which would be considered nonspecific. Heterogeneity of terminology is perhaps not surprising from the sleep medicine perspective, where terms like fatigue, sleepiness, hypersomnia, and fogginess may be used interchangeably by patients in clinical practice and may not be well distinguished even in research contexts.

Fourth, the sources differed in their listing of adverse effects and drug interactions.

\section{Safety considerations for natural remedies}

Natural remedies may be perceived as safer than prescriptions, although risk concerns have been raised for many supplements..$^{37,38}$ The six sources we assessed often included 
general suggestions and cautions regarding uncertainties surrounding the preparations, such as purity and standardization. Strikingly, a recent report indicated that the majority of herbal remedies sampled had contamination, substitution, or use of fillers not indicated in the labeling. ${ }^{39}$

Figures $\mathrm{S} 1$ and $\mathrm{S} 2$ contain adverse event listings from the PDR for Herbal Medicines and UpToDate. Because the safety of these agents has not been studied with rigor, the absence of reported risks should not be interpreted as a demonstration of safety. Many cautioned against use in pregnancy. Several remedies in the UpToDate listing for hepatic injury (often transient, but can be severe) are found in Figure 2: valerian, mistletoe, skullcap, and kava. Some common remedies had extensive detail of reported adverse risks, such as valerian, ginseng, ginko, and kava; readers are directed to the PDR for additional details. Other resources are also available in this regard. The NIH maintains a public database for searching for liver toxicity for drugs and supplements (http://www.livertox.nih.gov/). In addition, UpToDate recommends www.consumerlab.com, for checking which brands have undergone independent testing, and the FDA has a web resource for safety and recalls that includes foods and herbals (http://www.fda. gov/Safety/Recalls/).

\section{Pragmatic challenges of requiring conventional evidence standards for herbals}

The paucity of data for herbal therapy for insomnia is in principle addressable by randomized placebo controlled trials. However, the time horizon and resources required for such testing across even a subset of the remedies listed herein is daunting and seems unlikely to occur even with optimistic funding projections. The n-of- 1 trial approach, despite being an important innovation, is unlikely to solve this evidence problem, as it is still resource intensive and requires research protocols, in part due to the placebo component of the design. Yet, many patients continue to seek advice about or actively self-medicate with alternatives to prescription agents, indicating a need to bridge this evidence gap.

Providers may vary in their comfort level and risk tolerance in these situations. ${ }^{37,38}$ Moreover, individuals arguably consider their personal perspective over what is reported in RCTs. The disconnect between patients' views and those of some providers is illustrated by data suggesting patients were not talking to their providers about CAM, believed physicians did not know enough about CAM, and would continue to use CAM even if clinical trials showed lack of efficacy. ${ }^{40}$ Even for FDA-approved hypnotics, it seems unlikely that patients are anchoring their usage decisions upon the absolute number of extra minutes of sleep versus placebo from an RCT. Thus, guidance for how to think about their goals and leverage their diaries via a semiquantitative approach can be empowering, especially for patients who may be overwhelmed at their situation, finding it challenging to identify patterns or assess anything beyond a gestalt feeling in response to a new intervention.

\section{A practical clinical method for guided self-testing}

There are several motivating factors driving our approach, which are in line with common clinical practice when pharmacological or behavioral therapy trials are pursued. Selftracking with diaries is often standard in clinical practice, but how the entries drive decisions and actions remains a qualitative exercise. Any method should ideally promote certain patient care goals. Borrowing from the concept of statistical power from traditional group-wise clinical trials, the idea is to apply quantitative guidance to a single person measuring their sleep over multiple nights. Providing guidance even for simple questions like "how long should I try this?" can be structured, drawing from diary entries in a more quantitative manner, and incorporating the reality of night-to-night variability that is common in chronic insomnia. ${ }^{41-43}$ What specific aspects of sleep should be tracked is also highly personal, though diaries commonly inquire about sleep latency, number of awakenings, TST, etc.

We use patient-specific simple inputs to create personalized sleep strategies that can be applied to any chosen remedy to improve sleep. These personalized strategies use patient-specific goals as a form of power calculation and goal checking for self-testing. Figure 3 illustrates a visual algorithm for the process. The individual is first guided through the process of simplifying the many facets of sleep that could be tracked in principle into a binary label of "good" or "bad" sleep. This seemingly simple step overcomes two key challenges. First, having multiple sleep features involved in a treatment goal complicates self-testing by reducing power (i.e., prolonging the time needed to evaluate effects). Second, different patients attribute different importance to sleep features, which requires customization of diaries and of power calculations. Collapsing patient-determined factors into a binary outcome allows the approach to generalize across any patient-derived definition of "good" sleep and 


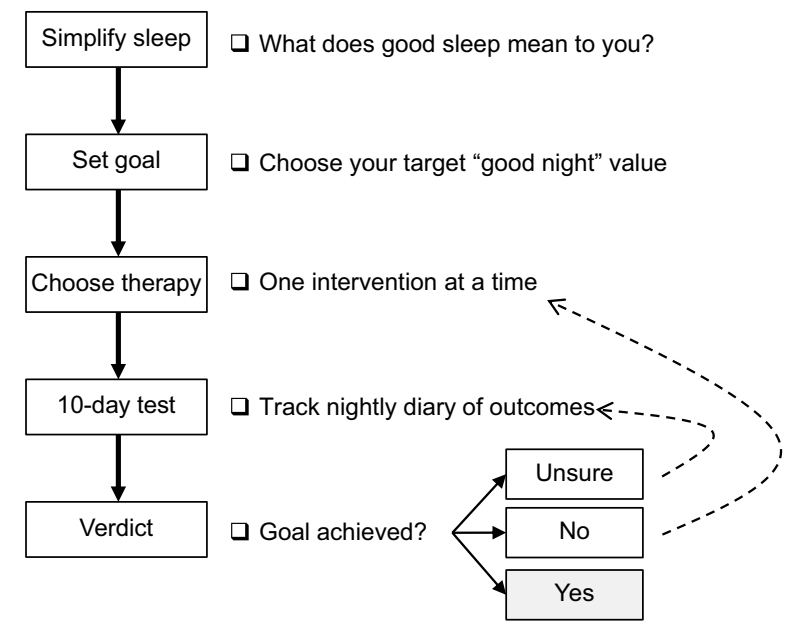

Figure 3 Flow chart describing the steps of guided self-testing. Note: Dotted lines indicate optional recursive paths depending on self-assessment of goal achievement.

allows any patient to participate in this guided process using a common end point: what is your goal percentage of good nights? This is akin to how composite end points are used in large RCTs or how "effect size" seeks to render different outcomes on a common scale.

Once the sleep goal and the remedy are chosen, an initial 10-night assessment is undertaken. The precise number of nights is less important than the implications of what value is chosen, as a trade-off between duration of the test, and resolution of probability of a good night. Figure 4 illustrates the challenges with assessing sleep with small numbers of observations. For a binary outcome (good vs bad night), if only one observation is made, the only observable proportion of good nights is 0 or $100 \%$ - neither is likely to be the true probability. For two nights, the only observable proportions are 0,50 , or $100 \%$ - also a poor resolution of probability. Brief trials, such as one to two nights, create two important inferential risks: 1) overconfidence (if the result happens to be $100 \%$ good) or 2) overpessimism (if the result happens to be $0 \%$ good). Consider a fair coin flipped twice, where heads indicates a good night, and tails indicates a bad night: there is a $50 \%$ chance that the observed two-flip outcome will be extreme ( $\mathrm{HH}$ and TT are each $25 \%$ chance) rather than the correct estimate of the true probability of 50\% (HT and TH are each $25 \%$ chance). With increasing number of observations, one increases the resolution of possible observed proportions of good nights. For 10 nights, the observable proportions have a more clinically interpretable coverage of intervals $(0,10,20 \%$, etc). Those wishing higher resolution would track themselves for additional nights (e.g., 20 nights for $5 \%$ intervals).

After the 10-night trial, the resulting observed percentage of good nights is compared to the binomial distribution of 10 "trials" under the assumption that the user-chosen goal probability of good nights has been achieved. If the observed percentage of good nights is within the acceptable "buffer" range, the remedy could be continued, assuming no adverse effects have occurred. For example, a patient might choose a goal of $70 \%$ good nights, while recognizing that any given 10-night period might have slightly more or slightly less observed good nights, even if the goal is met, that is, if 0.7 is the "true" probability. If the proportion is too far below the acceptable buffer, a new remedy can be tried (Figure 3, dotted arrow back to "choose therapy" step). If the value is close but not within the buffer range of the goal, then the individual has the option to pursue an additional 10 nights (Figure 3, dotted arrow back to "10-day test" step).

It is up to the individual to decide how much certainty they wish to achieve in estimating their probability of a good night. For any sequence of nights, the maximum likelihood estimate of the true probability of a good night is equal to the observed probability of a good night. The number of nights assessed to derive the observed probability will

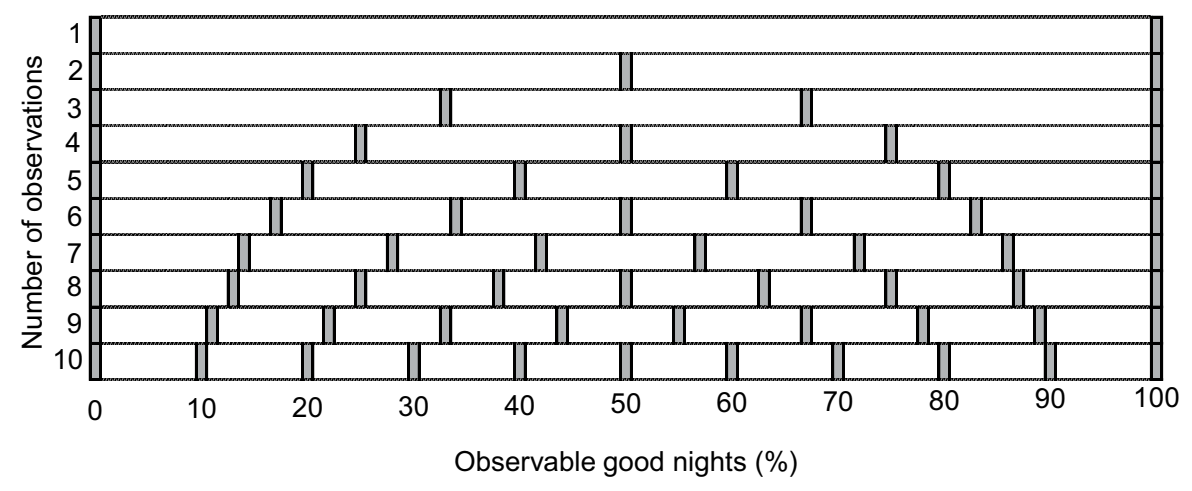

Figure 4 Observable proportions of good nights, assuming binary outcome (good vs bad) across a range of $1-10$ nights.

Notes: The $X$-axis is the percentage of good nights. The $Y$-axis is the number of nights in a trial of self-testing. The gray bars in each row indicate the possible observed percentage of good nights. For example, in a two-night trial, the only observable proportions are 0,50 , and $100 \%$ good nights. 

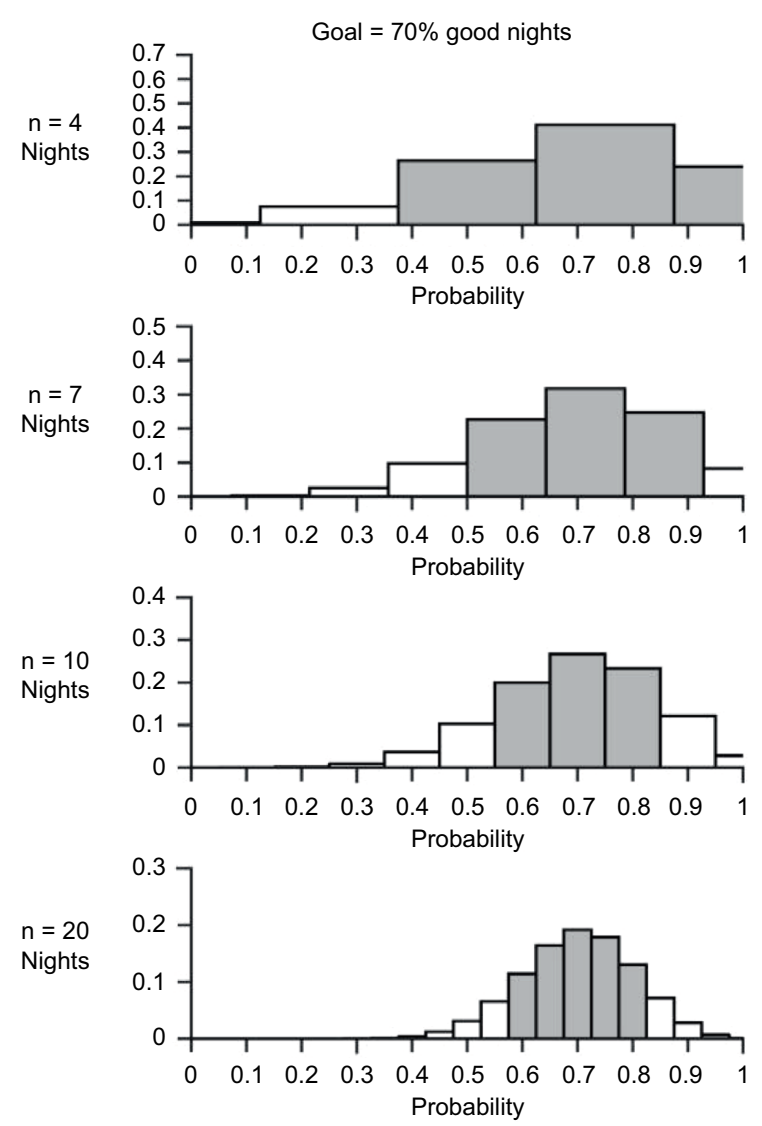
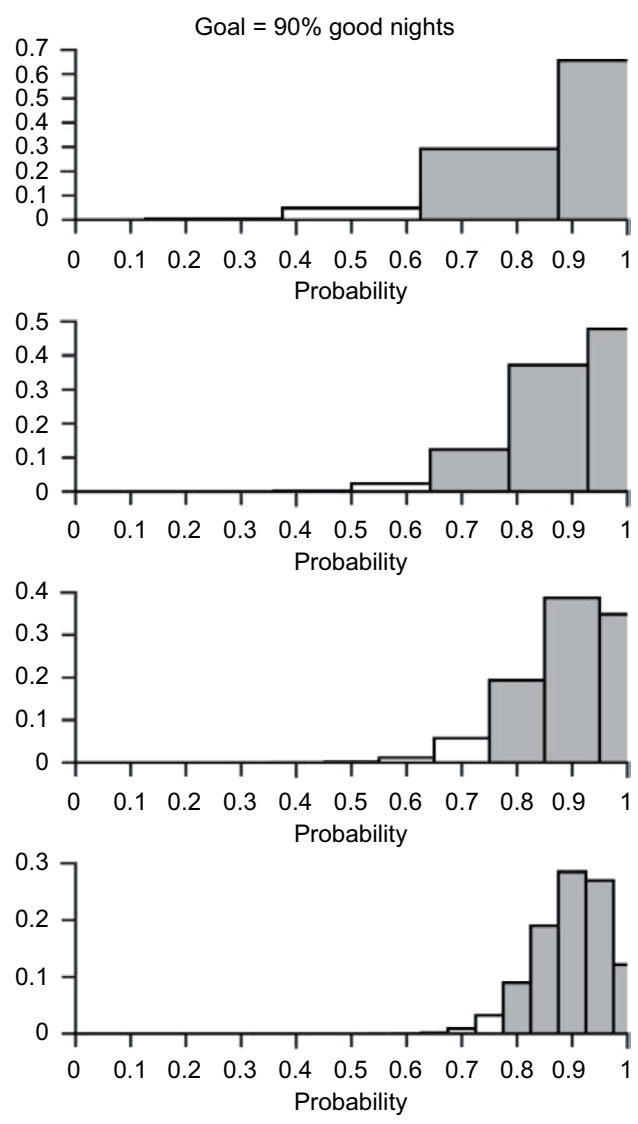

Figure 5 Histograms of binomial distributions across different true probabilities of a good night and different number of nights tested.

Notes: The binomial distribution is shown under the assumption of two self-testing goals: $70 \%$ chance of a good night (left column) and $90 \%$ chance of a good night (right column). For each of these goal proportions, the distribution is shown for different number of nights of assessment (4, 7, 10, and 20; rows). In each case, the gray bars indicate a buffer around the goal proportion that might be considered acceptably close; this assumed a $10 \%$ buffer on either side of the goal, but for four- and seven-night tests, the resolution does not allow thus, and hence the buffer extends to the next nearest histogram bin. By convention, bins are centered around the actual observed value, resulting in half-sized bins at the edges of 0 and I probability. The $y$-axes are fractions of occurrences in each panel.

shape how confident one is with that estimate. Figure 5 illustrates the binomial distribution for $n=4$ nights to $n=$ 20 nights (rows), for goal percentage of good nights of 70 or $90 \%$ (left and right columns). The gray shading indicates a range of "acceptable" buffer around the goal. This can be adjusted and personalized but is set here for illustration with a bound of $\pm 10 \%$ of the goal proportion of good nights. To summarize, our proposed approach has key advantages that draw directly from patient care and translate directly into clinical guidance.

1. Simplifying diary tracking to a common terminology (good vs bad) allows adaptation to any personal definition of what "good" sleep may be. This streamlines diary interpretation and allows the method to generalize. It does not preclude individuals tracking other details, akin to secondary or exploratory aims in a clinical trial.

2. The diary tracking feeds directly into the process. By linking diary tracking results directly with patient-driven decisions using basic statistical methods, the effort of self-tracking feeds directly into guided but flexible decision making.

3. There is no requirement for baseline diary pattern tracking, because the outcome is purely "goal" focused, rather than the typical clinical trial approach of showing a significant difference from pretreatment baseline values.

4. The method mitigates the risk of reacting to a single night, intuitively paralleling how we could not judge the fairness of a coin based on one toss. Instead of responding to "noise" of individual nights, it provides a context for patients to embrace their own nightly variability so it no longer represents a fear of uncertainty.

5. The results are by definition assured to be relevant to the individual. In this way, we can reconcile the realities of response heterogeneity in a chronic disease with highly individualized causes, contributors, and treatment goals.

Disadvantages of the method are also recognized. That the outcomes of sleep are forced into a simplified binary answer may strike some individuals as oversimplified in 
that they cannot comfortably render their complex issue into such a categorization. Certainly, multifactor outcomes can be modeled but require larger self-testing durations to analyze in this manner (akin to the standard practice of defining one outcome, often a composite outcome, in clinical trials, to establish trial power). Further, we recognize that the process does not engage any placebo role. However, the costs and infrastructure required to conduct placebo-controlled testing, which necessarily involves research protocols rather than routine clinical practice, are circumvented. Additionally, the individual's goals of therapy may not involve statistical proof of difference from placebo or statistical proof of difference from baseline - the two major goals of prospective randomized trials. Finally, the results of any individual's testing may not generalize beyond that individual. However, combining individualized results can inform future population studies, and a meta-analysis of n-of- 1 trials, for example, has been reported. ${ }^{33}$

\section{Conclusion}

The adaptive method naturally integrates with what already happens in a qualitative manner for patient self-assessments to provide a structure upon which already accepted clinical goals can be actively supported. The goal-oriented strategy of self-testing is distinct from the traditional statistical comparison of superiority of postintervention sleep versus baseline sleep, which may resonate less with patients who may have goals not well captured by traditional methods. Instead, the approach augments usual care (i.e., open label) of prescribing while remaining personalized. For patients and providers who wish to consider these agents after careful discussion and consideration of the uncertainties regarding safety and efficacy, such a system could provide a useful and practical clinical framework that serves multiple clinical goals.

\section{Acknowledgments}

We thank Jessica Kelly for assistance with early aspects of the literature review. Kate Romero and Balaji Goparaju are cofirst authors. This was not an industry-supported study. We thank Dr Suzanne Bertisch for useful discussions.

\section{Disclosure}

Dr Bianchi has received funding from the Department of Neurology, Massachusetts General Hospital, the Center for Integration of Medicine and Innovative Technology, the Milton Family Foundation, the MGH-MIT Grand Challenge, and the American Sleep Medicine Foundation. Dr Bianchi has a patent pending on a home sleep monitoring device. Dr
Bianchi has research contracts with MC10 and Insomnisolv, a consulting agreement with McKesson and International Flavors and Fragrances, received payment for educational material from Oakstone Publishing, and has provided expert testimony in sleep medicine. Dr Bianchi serves as a medical monitor for Pfizer. Dr Westover has received funding from the NIH (NIH-NINDS 1K23NS090900). The other authors report no conflicts of interest in this work.

\section{References}

1. Ohayon MM. Epidemiology of insomnia: what we know and what we still need to learn. Sleep Med Rev. 2002;6(2):97-111.

2. Schutte-Rodin S, Broch L, Buysse D, Dorsey C, Sateia M. Clinical guideline for the evaluation and management of chronic insomnia in adults. J Clin Sleep Med. 2008;4(5):487-504.

3. Espie CA, Luik AI, Cape J, et al. Digital cognitive behavioural therapy for insomnia versus sleep hygiene education: the impact of improved sleep on functional health, quality of life and psychological well-being. Study protocol for a randomised controlled trial. Trials. 2016;17(1):257.

4. van der Zweerde T, Lancee J, Slottje P, et al. Cost-effectiveness of i-Sleep, a guided online CBT intervention, for patients with insomnia in general practice: protocol of a pragmatic randomized controlled trial. BMC Psychiatry. 2016;16:85.

5. Lancee J, van Straten A, Morina N, Kaldo V, Kamphuis JH. Guided online or face-to-face cognitive behavioral treatment for insomnia: a randomized wait-list controlled trial. Sleep. 2016;39(1):183-191.

6. Cheng SK, Dizon J. Computerised cognitive behavioural therapy for insomnia: a systematic review and meta-analysis. Psychother Psychosom. 2012;81(4):206-216.

7. Lader M. Benzodiazepines revisited - will we ever learn? Addiction. 2011;106(12):2086-2109.

8. Kripke DF, Langer RD, Kline LE. Hypnotics' association with mortality or cancer: a matched cohort study. BMJ Open. 2012;2(1):e00850.

9. Bianchi MT, Thomas RJ, Ellenbogen JM. Hypnotics and mortality risk. J Clin Sleep Med. 2012;8(4):351-352.

10. Kripke DF. Hypnotic drug risks of mortality, infection, depression, and cancer: but lack of benefit. F1000Res. 2016;5:918.

11. Antai-Otong D. The art of prescribing. Risks and benefits of nonbenzodiazepine receptor agonists in the treatment of acute primary insomnia in older adults. Perspect Psychiatr Care. 2006;42(3):196-200.

12. Glass J, Lanctot KL, Herrmann N, Sproule BA, Busto UE. Sedative hypnotics in older people with insomnia: meta-analysis of risks and benefits. BMJ. 2005;331(7526):1169.

13. Allain H, Bentue-Ferrer D, Polard E, Akwa Y, Patat A. Postural instability and consequent falls and hip fractures associated with use of hypnotics in the elderly: a comparative review. Drugs Aging. 2005;22(9):749-765.

14. Mason M, Cates CJ, Smith I. Effects of opioid, hypnotic and sedating medications on sleep-disordered breathing in adults with obstructive sleep apnoea. Cochrane Database Syst Rev. 2015;(7):CD011090.

15. Yang C, White DP, Winkelman JW. Antidepressants and periodic leg movements of sleep. Biol Psychiatry. 2005;58(6):510-514.

16. Eckert DJ, Malhotra A, Wellman A, White DP. Trazodone increases the respiratory arousal threshold in patients with obstructive sleep apnea and a low arousal threshold. Sleep. 2014;37(4):811-819.

17. Buscemi N, Vandermeer B, Friesen C, et al. The efficacy and safety of drug treatments for chronic insomnia in adults: a meta-analysis of RCTs. J Gen Intern Med. 2007;22(9):1335-1350.

18. Brzezinski A, Vangel MG, Wurtman RJ, et al. Effects of exogenous melatonin on sleep: a meta-analysis. Sleep Med Rev. 2005;9(1):41-50.

19. Buscemi N, Vandermeer B, Hooton N, et al. The efficacy and safety of exogenous melatonin for primary sleep disorders. A meta-analysis. J Gen Intern Med. 2005;20(12):1151-1158.

20. Bent S, Padula A, Moore D, Patterson M, Mehling W. Valerian for sleep: a systematic review and meta-analysis. Am JMed. 2006;119(12):1005-1012. 
21. Fernandez-San-Martin MI, Masa-Font R, Palacios-Soler L, SanchoGomez P, Calbo-Caldentey C, Flores-Mateo G. Effectiveness of Valerian on insomnia: a meta-analysis of randomized placebo-controlled trials. Sleep Med. 2010;11(6):505-511.

22. Leach MJ, Page AT. Herbal medicine for insomnia: a systematic review and meta-analysis. Sleep Med Rev. 2015;24:1-12.

23. Sarris J, Byrne GJ. A systematic review of insomnia and complementary medicine. Sleep Med Rev. 2011;15(2):99-106.

24. Kaufman DW, Kelly JP, Rosenberg L, Anderson TE, Mitchell AA. Recent patterns of medication use in the ambulatory adult population of the United States: the Slone survey. JAMA. 2002;287(3): 337-344.

25. Clarke TC, Black LI, Stussman BJ, Barnes PM, Nahin RL. Trends in the use of complementary health approaches among adults: United States, 2002-2012. Natl Health Stat Report. 2015;(79):1-16.

26. Tindle HA, Davis RB, Phillips RS, Eisenberg DM. Trends in use of complementary and alternative medicine by US adults: 1997-2002. Altern Ther Health Med. 2005;11(1):42-49.

27. Pearson NJ, Johnson LL, Nahin RL. Insomnia, trouble sleeping, and complementary and alternative medicine: analysis of the 2002 national health interview survey data. Arch Intern Med. 2006;166(16): 1775-1782.

28. Wells RE, Phillips RS, Schachter SC, McCarthy EP. Complementary and alternative medicine use among US adults with common neurological conditions. J Neurol. 2010;257(11):1822-1831.

29. Bertisch SM, Wee CC, Phillips RS, McCarthy EP. Alternative mindbody therapies used by adults with medical conditions. J Psychosom Res. 2009;66(6):511-519.

30. Bertisch SM, Wells RE, Smith MT, McCarthy EP. Use of relaxation techniques and complementary and alternative medicine by American adults with insomnia symptoms: results from a national survey. $J$ Clin Sleep Med. 2012;8(6):681-691.
31. Duan N, Kravitz RL, Schmid CH. Single-patient (n-of-1) trials: a pragmatic clinical decision methodology for patient-centered comparative effectiveness research. J Clin Epidemiol. 2013;66(8 Suppl):S21-S28.

32. Lillie EO, Patay B, Diamant J, Issell B, Topol EJ, Schork NJ. The n-of-1 clinical trial: the ultimate strategy for individualizing medicine? Per Med. 2011;8(2):161-173.

33. Punja S, Bukutu C, Shamseer L, et al. N-of-1 trials are a tapestry of heterogeneity. J Clin Epidemiol. 2016;76:47-56.

34. Gruenwald J, Brendler T, Jaenicke C, editors. PDR for Herbal Medicines. 4th ed. Montvale, NJ: Thomson Healthcare, Inc.; 2007.

35. Chevallier A. Encyclopedia of Herbal Medicine. 2nd ed. New York, NY: DK; 2016.

36. The Drug \& Natural Medicine Advisor. Richmond, VA: Time-Life Books; 2002.

37. Ashar BH, Rowland-Seymour A. Advising patients who use dietary supplements. Am J Med. 2008;121(2):91-97.

38. Glisson JK, Walker LA. How physicians should evaluate dietary supplements. Am J Med. 2010;123(7):577-582.

39. Newmaster SG, Grguric M, Shanmughanandhan D, Ramalingam S, Ragupathy S. DNA barcoding detects contamination and substitution in North American herbal products. BMC Med. 2013;11:222.

40. Blendon RJ, DesRoches CM, Benson JM, Brodie M, Altman DE. Americans' views on the use and regulation of dietary supplements. Arch Intern Med. 2001;161(6):805-810.

41. Baron KG, Reid KJ, Malkani RG, Kang J, Zee PC. Sleep variability among older adults with insomnia: associations with sleep quality and cardiometabolic disease risk. Behav Sleep Med. 2017;15(2):144-157.

42. Buysse DJ, Cheng Y, Germain A, et al. Night-to-night sleep variability in older adults with and without chronic insomnia. Sleep Med. 2010; 11(1):56-64.

43. Suh S, Nowakowski S, Bernert RA, et al. Clinical significance of nightto-night sleep variability in insomnia. Sleep Med. 2012;13(5):469-475. 


\section{Supplementary materials}

\begin{tabular}{|c|c|}
\hline Remedy & Risks (from PDR and UpToDate) \\
\hline 5-HTP & Avoid with carbidopa, antidepressants \\
\hline \multicolumn{2}{|l|}{ Albizia julibrissin } \\
\hline Angelica archangelica & UV sensitivity; blood thinning \\
\hline Anise (Pimpinella) & Blood thinning \\
\hline \multicolumn{2}{|l|}{ Ashwagandha } \\
\hline \multicolumn{2}{|l|}{ Betony } \\
\hline Bitter Orange (Neroli) & UV sensitivity \\
\hline \multicolumn{2}{|l|}{ Black Horehound } \\
\hline \multicolumn{2}{|l|}{ Black nightshade } \\
\hline Bog Bean & Avoid in GI diseases \\
\hline Borage & GI upset; avoid in epilepsy and schizophrenia \\
\hline Bugleweed & Avoid in thyroid disease \\
\hline Butterbur & Must have alkaloid-free preparation (carcinogenic; hepatotoxic) \\
\hline \multicolumn{2}{|l|}{ California Poppy } \\
\hline \multicolumn{2}{|l|}{ Catnip (Nepeta) } \\
\hline \multicolumn{2}{|l|}{ Catuaba } \\
\hline Celery seed & Avoid in renal disease; photosensitivity; hypoglycemic \\
\hline Chamomile (German) & Blood thinning \\
\hline Chaste Tree Berry & Gl upset; dizziness; confusion; itching; avoid with dopaminergics \\
\hline \multicolumn{2}{|l|}{ Corn Poppy } \\
\hline \multicolumn{2}{|l|}{ Cowslip } \\
\hline Cypress & Possible renal effects at high dose \\
\hline \multicolumn{2}{|l|}{ Dan-shen (Chinese red sage) } \\
\hline Dong Quai (Angelica sinensis) & GI upset; blood thinning; UV sensitivity \\
\hline \multicolumn{2}{|l|}{ False Schisandra } \\
\hline \multicolumn{2}{|l|}{ Fo-ti } \\
\hline \multicolumn{2}{|l|}{ Gardenia } \\
\hline Ginkgo biloba & Numerous, including seizure; blood thinning; GI upset; multiple drug-interactions \\
\hline Ginseng (Panax) & Numerous AEs \& drug interactions; altered blood sugar; estrogenic; hypertension; cardioactive \\
\hline \multicolumn{2}{|l|}{ Ginseng (Siberian) } \\
\hline Gotu Kola & UV sensitivity; blood sugar and lipid increase; infertility \\
\hline \multicolumn{2}{|l|}{ Hawthorn } \\
\hline \multicolumn{2}{|l|}{ Heather } \\
\hline Hops & Avoid in depression, breast cancer; rare hematologic and anaphylactic \\
\hline \multicolumn{2}{|l|}{ Ignatia } \\
\hline Jamaican Dogwood & \\
\hline Jasmine & \\
\hline
\end{tabular}

Figure SI Natural remedies (alphabetical, A-J), with risks as summarized by two sources (Herbal Medicine PDR, and UpToDate).

Abbreviations: AEs, adverse effects; GI, gastro-intestinal; PDR, Physician's Desk Reference for Herbal Medicines; UV, ultraviolet light. 


\begin{tabular}{|c|c|}
\hline Remedy & Risks (from PDR and UpToDate) \\
\hline \multicolumn{2}{|l|}{ Jatamansi } \\
\hline Kava & Depression; dyskinesia; liver toxicity; numerous others and drug interactions \\
\hline Lavender (English) & (none for aroma; do not ingest) \\
\hline \multicolumn{2}{|l|}{ Lemon Balm } \\
\hline \multicolumn{2}{|l|}{ Lemon Verbena } \\
\hline \multicolumn{2}{|l|}{ Lemon-Wood (Schisandra) } \\
\hline \multicolumn{2}{|l|}{ Linden } \\
\hline Male Fern & Gl upset; avoid if diabetic, anemic, cardiac or hepati or renal disease \\
\hline Meliltot (sweet clover) & Liver enzyme elevations \\
\hline \multicolumn{2}{|l|}{ Mugwort } \\
\hline \multicolumn{2}{|l|}{ Nerve Root (Lady's Slipper) } \\
\hline \multicolumn{2}{|l|}{ Nutmeg (and Mace) } \\
\hline Oats (Avena sativa) & Avoid in gluten sensitivity; reduced statin absorption; possible increase triglycerides \\
\hline \multicolumn{2}{|l|}{ Pasque Flower } \\
\hline \multicolumn{2}{|l|}{ Passion Flower } \\
\hline Peppermint & Avoid in acid reflux, gall bladder \& liver disease; reduces CCB effects; blocks CYP1A2, CYP2E \\
\hline Poppyseed & Multiple AEs \\
\hline Rauwolfia (serpentwood) & Avoid in depression, pheochromocytoma, ulcers; CNS activity; avoid w/digitalis \\
\hline \multicolumn{2}{|l|}{ Red-Spur Valerian } \\
\hline \multicolumn{2}{|c|}{ Rehmannia (Chinese foxglove) } \\
\hline \multicolumn{2}{|l|}{ Reishi Mushroom } \\
\hline Rhodiola & Avoid in bipolar \\
\hline \multicolumn{2}{|l|}{ Saffron } \\
\hline Schisandra (Wu-Wei-Zi) & Gl upset; avoid in epilepsy, hypertension or intracranial pressure \\
\hline \multicolumn{2}{|l|}{ Senburi } \\
\hline Skullcap & Liver injury \\
\hline St. John's Wort & Numerous AEs and drug interactions; lead contamination \\
\hline \multicolumn{2}{|l|}{ Sumbul } \\
\hline Sweet Vernal Grass & Hepatic injury; blood thinning \\
\hline \multicolumn{2}{|l|}{ Sweet Violet } \\
\hline Sweet Woodruff & Hepatic injury; blood thinning \\
\hline \multicolumn{2}{|l|}{ Tarragon } \\
\hline Valerian & Numerous AEs and drug interactions; liver injury \\
\hline \multicolumn{2}{|l|}{ Water Hyssop } \\
\hline \multicolumn{2}{|l|}{ Wild Lettuce } \\
\hline Yarrow & Infertility; avoid with iron supplements; trace thujone contamination \\
\hline \multicolumn{2}{|l|}{ Ylang Ylang oil } \\
\hline Zyzyphus (Da-Zao) & Possible liver injury \\
\hline
\end{tabular}

Figure S2 Natural remedies (alphabetical, J-Z), with risks as summarized by two sources (Herbal Medicine PDR, and UpToDate).

Abbreviations: AEs, adverse effects; CCB, calcium channel blocker; CNS, central nervous system; GI, gastro-intestinal; PDR, Physician's Desk Reference for Herbal Medicines. 
Nature and Science of Sleep is an international, peer-reviewed, open access journal covering all aspects of sleep science and sleep medicine, including the neurophysiology and functions of sleep, the genetics of sleep, sleep and society, biological rhythms, dreaming, sleep disorders and therapy, and strategies to optimize healthy sleep. The manuscript management system is completely online and includes a very quick and fair peer-review system, which is all easy to use. Visit http://www. dovepress.com/testimonials.php to read real quotes from published authors.

Submit your manuscript here: https://www.dovepress.com/nature-and-science-of-sleep-journal 